\title{
Analysis of Exact Linearization and Aproximate Feedback Linearization Techniques
}

\author{
Gildeberto S. Cardoso ${ }^{1}$ and Leizer Schnitman ${ }^{2}$ \\ ${ }^{1}$ Universidade Federal do Recôncavo da Bahia, Rua Rui Barbosa, No. 710, \\ CEP 44.380-000—Cruz das Almas, BA, Brazil \\ 2 Post Graduate Program in Mechatronics, Universidade Federal da Bahia, \\ Rua Aristides Novis No. 2, Federação, CEP 40.210-630—Salvador, BA, Brazil \\ Correspondence should be addressed to Gildeberto S. Cardoso, gildeberto@ufrb.edu.br
}

Received 19 October 2010; Accepted 3 March 2011

Academic Editor: Bin Liu

Copyright (C) 2011 G. S. Cardoso and L. Schnitman. This is an open access article distributed under the Creative Commons Attribution License, which permits unrestricted use, distribution, and reproduction in any medium, provided the original work is properly cited.

This paper presents a study of linear control systems based on exact feedback linearization and approximate feedback linearization. As exact feedback linearization is applied, a linear controller can perform the control objectives. The approximate feedback linearization is required when a nonlinear system presents a noninvolutive property. It uses a Taylor series expansion in order to compute a nonlinear transformation of coordinates to satisfy the involutivity conditions.

\section{Introduction}

The control theory of nonlinear system has been receiving increasing attention in recent years, both for its technical importance as well as for its impact in various fields of application. In strategic areas, such as aerospace, chemical and petrochemical industries, bioengineering, and robotics, a new practical application for this tool appears every day.

System nonlinearity is characterized when at least one component or subsystem is nonlinear [1]. The classic methods used in the study of nonlinear systems, particularly frequency analysis, are not usually applied to the nonlinear systems. It is necessary to use other methods to study the control of these systems.

When the nonlinear effects of systems become significant, nonlinear control techniques generally fail to produce the desired performance. In these cases, there are several nonlinear control techniques that have proven to supply better results.

Among the possible techniques used to deal with the nonlinear control problem, this paper studies the approximate feedback linearization technique for controlling nonlinear systems. The applications of these techniques are illustrated through some examples. 


\section{Analysis of Necessary and Sufficient Conditons for Exact Feedback Linearization}

The objective of this section is to detail the necessary and sufficient conditions for inputstate linearization. These conditions are limiting to the implementation of the exact feedback linearization technique. However, as a solution to this problem, the approximate linearization technique will be described.

Initially, we consider a single input $u(t)$ for a nonlinear system, given by

$$
\dot{\mathbf{x}}=\mathbf{f}(\mathbf{x})+\mathbf{g}(\mathbf{x}) u
$$

This system is known as linear in the control and linear in the input, among other denominations. The functions $\mathbf{f}(\mathbf{x})$ and $\mathbf{g}(\mathbf{x})$ are $C^{\infty}$ vectors with field in $\dot{\mathbf{x}} \in R^{n}$.

The system in the form (2.1) is input-state linearizable if there is a region $\Omega$ in $R^{n}$ that meets the following conditions:

(i) the vector field $\left\{\mathbf{g}, a d_{\mathrm{f}} \mathbf{g}, \ldots, \mathbf{a d}_{\mathbf{f}}^{n-1} \mathbf{g}\right\}$ is linearly independents in $\Omega$,

(ii) the set of vectors $\left[a d_{\mathrm{f}}^{i} \mathbf{g}, a d_{\mathrm{f}}^{j} \mathbf{g}\right]=\operatorname{span}\left\{\mathbf{g}, a d_{\mathrm{f}} \mathbf{g}, \ldots, a d_{\mathrm{f}}^{n-2} \mathbf{g}\right\}$ for $i, j=0,1,2, \ldots, n-2$ is involutive,

where $\left[a d_{\mathrm{f}}^{i} \mathbf{g}, a d_{\mathrm{f}}^{j} \mathbf{g}\right]$ indicates the Lie bracket and $a d_{\mathrm{f}}^{i} \mathbf{g}$ is defined as [2]:

$$
a d_{\mathrm{f}}^{i} \mathbf{g}=\left[\mathbf{g}, a d_{\mathrm{f}}^{i-1} \mathbf{g}\right]
$$

The first condition indicates the system controllability in which the vector fields $\left\{\mathbf{g}, a d_{\mathbf{f}} \mathbf{g}, \cdots, a d_{\mathbf{f}}^{n-1} \mathbf{g}\right\}$ are equivalent to the controllability matrix for linear systems $\left[\begin{array}{lllll}\mathbf{b} & \mathbf{A b} & \mathbf{A}^{2} \mathbf{b} & \cdots & \mathbf{A}^{n-1} \mathbf{b}\end{array}\right][1]$.

The second involutivity condition enables to find out a new vector of linear state through the states feedback.

\section{Exact Feedback Linearization}

If the aforementioned conditions were satisfied, then it is possible to find a scalar function $\phi(\mathbf{x})$ such that

$$
\begin{gathered}
\frac{\partial \phi(\mathbf{x})}{\partial \mathbf{x}} a d_{\mathbf{f}}^{i} \mathbf{g}=0, \quad i=0,1,2, \ldots, n-2, \\
\frac{\partial \phi(\mathbf{x})}{\partial \mathbf{x}} a d_{\mathbf{f}}^{n-1} \mathbf{g} \neq 0
\end{gathered}
$$

where $\phi(\mathbf{x})$ defines a state transformation $\mathbf{z}$ given by [1]

$$
\mathbf{z}=T(\mathbf{x})=\left[\begin{array}{llll}
L_{\mathrm{f}}^{0} \phi(\mathbf{x}) & L_{\mathrm{f}}^{1} \phi(\mathbf{x}) & \cdots & L_{\mathrm{f}}^{n-1} \phi(\mathbf{x})
\end{array}\right] .
$$




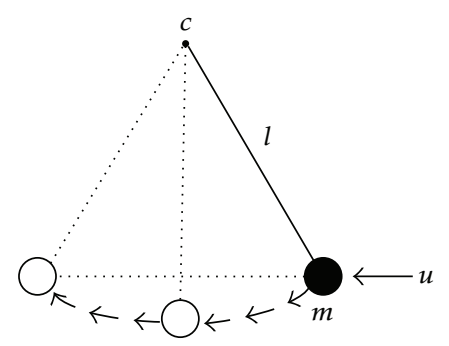

Figure 1: Simple pendulum.

A control input is then obtained through

$$
u=\alpha(\mathbf{x})+\beta(\mathbf{x}) v=\frac{-L_{\mathrm{f}}^{n} \phi(\mathbf{x})}{L_{\mathrm{g}} L_{\mathrm{f}}^{n-1} \phi(\mathbf{x})}+\frac{1}{L_{\mathrm{g}} L_{\mathrm{f}}^{n-1} \phi(\mathbf{x})} v,
$$

such that the closed-loop system has a new set of variables in linear state

$$
\dot{\mathbf{z}}=\mathbf{A z}+\mathbf{b} v,
$$

where

$$
\mathbf{A}=\left[\begin{array}{ccc}
010 & \cdots & 0 \\
001 & \cdots & 0 \\
\cdots & \cdots & \cdots \\
000 & \cdots & 1 \\
000 & \cdots & 0
\end{array}\right], \quad \mathbf{b}=\left[\begin{array}{c}
0 \\
0 \\
\cdots \\
0 \\
1
\end{array}\right]
$$

$\mathbf{A}$ and $\mathbf{b}$ are in the canonical form of Brunovski however, there is no loss of generality; as every linear system may be written in the companion form by a state transformation [1].

\subsection{Inverted Pendulum Linearization}

Figure 1 shows an overview of a single pendulum. The pendulum may be described as a fixed end connected to a sphere through an inflexible rod and can be swung when stimulated by a force $u$ or has a nonzero initial condition.

The model of a pendulum is given by

$$
\begin{gathered}
\dot{x}_{1}=x_{2}, \\
\dot{x}_{2}=-\frac{g}{l} \sin \left(x_{1}\right)-\frac{b}{m} x_{2}+\frac{1}{m l} u,
\end{gathered}
$$

where $g, l, m, b, x_{1}$, and $x_{2}$ are, respectively, gravitational acceleration, length of rod, mass of the sphere, friction coefficient, rotation angle, and angular speed. 


\subsubsection{Calculation of Controllability and Involutivity of the Simple Pendulum}

As it is a second-order system, the vector field for verifying the controllability will be formed by $\left\{\mathbf{g}, a d_{\mathrm{f}} \mathbf{g}\right\}$, where,

$$
\begin{gathered}
\mathbf{g}=\left[\begin{array}{c}
0 \\
\frac{1}{m l}
\end{array}\right], \\
a d_{\mathbf{f}} \mathbf{g}=\left[\begin{array}{ll}
0 & 0 \\
0 & 0
\end{array}\right]\left[\begin{array}{c}
x_{2} \\
-\frac{g}{l} \sin \left(x_{1}\right)-\frac{b}{m} x_{2}
\end{array}\right]-\left[\begin{array}{ccc}
0 & 1 \\
-\frac{g}{l} \cos \left(x_{1}\right) & -\frac{b}{m}
\end{array}\right]\left[\begin{array}{c}
0 \\
1 \\
\frac{m}{m l}
\end{array}\right]=\left[\begin{array}{c}
-\frac{1}{m l} \\
-\frac{b}{m^{2} l}
\end{array}\right] .
\end{gathered}
$$

Hence, the vector field $\left\{\mathbf{g}, a d_{\mathbf{f}} \mathbf{g}\right\}$ will be given by

$$
\left\{\mathbf{g}, a d_{\mathrm{f}} \mathbf{g}\right\}=\left[\begin{array}{cc}
0 & -\frac{1}{m l} \\
\frac{1}{m l} & -\frac{b}{m^{2} l}
\end{array}\right] .
$$

The vector fields are linearly independent; therefore, the simple pendulum is controllable.

In order to calculate the involutivity of the system, it is necessary to calculate the Lie bracket of $\left\{\mathbf{g}, a d_{\mathbf{f}} \mathbf{g}\right\}$ and verify if it may be written as a linear combination of $\mathbf{g}$ and $a d_{\mathbf{f}} \mathbf{g}$

$$
\left[\mathbf{g}, a d_{\mathbf{f}} \mathbf{g}\right]=\left[\begin{array}{l}
0 \\
0 \\
0 \\
0
\end{array}\right] .
$$

The Lie bracket of $\left\{\mathbf{g}, a d_{\mathbf{f}} \mathbf{g}\right\}$ is equal to zero, as the column vectors of the matrix are constant. Therefore, the system is involutive, which satisfy the conditions to apply input-state linearization.

\subsubsection{Calculation of the New State Vector and the Input Variable Using Differential Geometry}

The first component of the state vector $T_{1}$ should be obtained through the expression (3.1). As the order of the system is equal to 2 , equation $\partial T_{1}$ becomes

$$
\left[\begin{array}{ll}
\frac{\partial T_{1}}{\partial x_{1}} & \frac{\partial T_{1}}{\partial x_{2}}
\end{array}\right]\left[\begin{array}{c}
0 \\
\frac{1}{m l}
\end{array}\right]=0
$$


resulting in

$$
\frac{1}{m l} \frac{\partial T_{1}}{\partial x_{2}}=0
$$

Therefore,

$$
\frac{\partial T_{1}}{\partial x_{2}}=0
$$

From (3.2),

$$
\left[\begin{array}{ll}
\frac{\partial T_{1}}{\partial x_{1}} & \frac{\partial T_{1}}{\partial x_{2}}
\end{array}\right]\left[\begin{array}{c}
-\frac{1}{m l} \\
-\frac{b}{m^{2} l}
\end{array}\right] \neq 0
$$

solving

$$
-\frac{1}{m l} \frac{\partial T_{1}}{\partial x_{1}} \neq 0
$$

Therefore,

$$
\frac{\partial T_{1}}{\partial x_{1}} \neq 0
$$

In order to satisfy the last equation, $T_{1}=x_{1}$ has to be chosen.

We use (3.3) to compute the second component of the new state vector $T_{2}$

$$
\begin{aligned}
T_{2} & =L_{\mathrm{f}} T_{1}=\left[\frac{\partial T_{1}}{\partial x_{1}}\right]\left[\begin{array}{c}
x_{2} \\
-\frac{\mathbf{g}}{l} \sin \left(x_{1}\right)-\frac{b}{m} x_{2}
\end{array}\right] \\
& =\left[\begin{array}{ll}
1 & 0
\end{array}\right]\left[\begin{array}{c}
x_{2} \\
-\frac{\mathbf{g}}{l} \sin \left(x_{1}\right)-\frac{b}{m} x_{2}
\end{array}\right]=x_{2} .
\end{aligned}
$$

This gives the new state vector

$$
\mathbf{T}=\left[\begin{array}{ll}
T_{1} & T_{2}
\end{array}\right]=\left[\begin{array}{ll}
x_{1} & x_{2}
\end{array}\right]
$$

The new input variable is calculated from (3.4), where

$$
\begin{gathered}
\alpha(\mathbf{x})=\frac{-(g / l) \sin \left(x_{1}\right)-(b / m) x_{2}}{1 / m l}=m g \sin \left(x_{1}\right)+l b x_{2}, \\
\beta(\mathbf{x})=-\frac{1}{1 / m l}=-m l .
\end{gathered}
$$




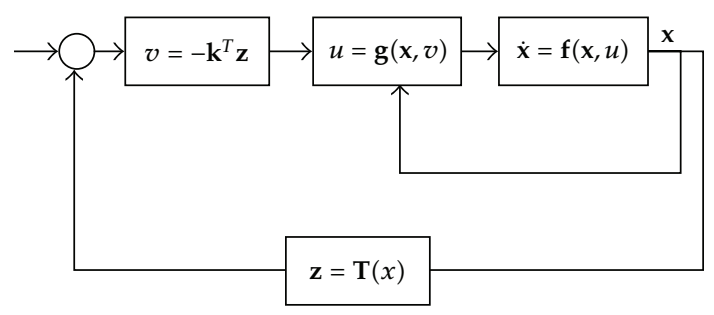

Figure 2: Control structure.

Therefore,

$$
u=m g \sin \left(x_{1}\right)+l b x_{2}-m l v \text {. }
$$

This results in a linear system

$$
\left[\begin{array}{l}
\dot{T}_{1} \\
\dot{T}_{2}
\end{array}\right]=\left[\begin{array}{c}
T_{2} \\
-v
\end{array}\right] .
$$

The system block diagram may be represented as shown in Figure 2.

The control structure, according to Figure 2, has two feedbacks. The first feedback is responsible for system linearization, eliminating the existing linearity. The second feedback is the controller project based on the state feedback.

\subsection{The Need for Differential Geometry to Calculate the Exact Linearization}

The linearization result shown previously might be obtained directly from

$$
u=\frac{1}{\mathbf{g}(\mathbf{x})}(-\mathbf{f}(\mathbf{x})+v)
$$

When this procedure is applied, the linearization calculation would be significantly less. The problem occurs when the control law obtained by this means does not permit the exact linearization.

As an example of the aforementioned problem, consider a nonlinear system given by

$$
\begin{gathered}
\mathbf{f}(\mathbf{x})=\left[\begin{array}{c}
-3 x_{1}+2 x_{2}+e^{x_{1}} \\
-x_{2} e^{x_{1}}
\end{array}\right], \\
\mathbf{g}(\mathbf{x})=\left[\begin{array}{l}
0 \\
x_{1}
\end{array}\right] .
\end{gathered}
$$


Applying (3.22), we will obtain:

$$
u=\frac{1}{x_{1}}\left(x_{2} e^{x_{1}}+v\right)
$$

This result does not make equation (3.23) a linear system. It requires a variable transformation by inference or by differential geometry. An approach through differential geometry is more generic and can be implemented in software through simple programming.

\subsubsection{The Use of Differential Geometry to Linearize the Previous Example}

The first component of the state vector $T(\mathbf{x})$ is obtained through

$$
\nabla T_{1}(\mathbf{x})=a d_{\mathbf{f}(\mathbf{x})}^{0} \mathbf{g}(\mathbf{x})=0
$$

As $a d_{\mathbf{f}(\mathbf{x})}^{0} \mathbf{g}(\mathbf{x})=\left[\begin{array}{c}0 \\ x_{1}\end{array}\right]$, we will have

$$
\left[\begin{array}{ll}
\frac{\partial T_{1}(\mathbf{x})}{\partial x_{1}} & \frac{\partial T_{1}(\mathbf{x})}{\partial x_{2}}
\end{array}\right]\left[\begin{array}{l}
0 \\
x_{1}
\end{array}\right]=0
$$

Therefore,

$$
\frac{\partial T_{1}(\mathbf{x})}{\partial x_{2}}=0
$$

If $a d_{\mathbf{f}(\mathbf{x})} \mathbf{g}(\mathbf{x})=\left[\begin{array}{c}-2 x_{1} \\ 3 x_{1}+2 x_{2}+e^{x_{1}}+x_{1} e^{x_{1}}\end{array}\right]$, obtained through the expression (3.2), then

$$
\left[\begin{array}{ll}
\frac{\partial T_{1}(\mathbf{x})}{\partial x_{1}} & \frac{\partial T_{1}(\mathbf{x})}{\partial x_{2}}
\end{array}\right]\left[\begin{array}{c}
-2 x_{1} \\
-3 x_{1}+2 x_{2}+e^{x_{1}}+x_{1} e^{x_{1}}
\end{array}\right] \neq 0 .
$$

Substituting the result of (3.28) in the matrix $\nabla T_{1}(\mathbf{x})$, we obtain

$$
\left[\begin{array}{ll}
\frac{\partial T_{1}(\mathbf{x})}{\partial x_{1}} & 0
\end{array}\right]\left[\begin{array}{c}
-2 x_{1} \\
-3 x_{1}+2 x_{2}+e^{x_{1}}+x_{1} e^{x_{1}}
\end{array}\right] \neq 0 .
$$

Consequently,

$$
-2 x_{1} \frac{\partial T_{1}(\mathbf{x})}{\partial x_{1}} \neq 0
$$

This expression indicates that $\partial T_{1}(\mathbf{x}) / \partial x_{1} \neq 0$; therefore, we should choose the new state $T_{1}(\mathbf{x})$ in such way that it satisfies this condition. 


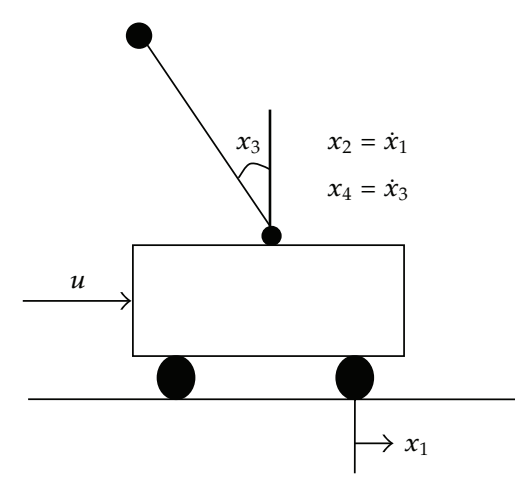

Figure 3: Inverted pendulum.

Choosing $T_{1}(\mathbf{x})=x_{1}$, we obtain

$$
\begin{aligned}
T_{2}(\mathbf{x}) & =L_{\mathbf{f}} T_{1}(\mathbf{x})=\nabla T_{1}(\mathbf{x})\left[\begin{array}{c}
-3 x_{1}+2 x_{2}+e^{x_{1}} \\
-x_{2} e^{x_{1}}
\end{array}\right] \\
& =\left[\begin{array}{ll}
1 & 0
\end{array}\right]\left[\begin{array}{c}
-3 x_{1}+2 x_{2}+e^{x_{1}} \\
-x_{2} e^{x_{1}}
\end{array}\right]=-3 x_{1}+2 x_{2}+e^{x_{1}} .
\end{aligned}
$$

With the new state space given by $\mathbf{z}=\mathbf{T}(\mathbf{x})$,

$$
\mathbf{z}=\mathbf{T}(\mathbf{x})=\left[\begin{array}{l}
T_{1}(\mathbf{x}) \\
T_{2}(\mathbf{x})
\end{array}\right]=\left[\begin{array}{c}
x_{1} \\
-3 x_{1}+2 x_{2}+e^{x_{1}}
\end{array}\right]
$$

\section{Approximate Feedback Linearization}

There are situations in the design of controllers where the nonlinear terms are significant to the extent that failure to consider these terms in the system model could cause it to perform badly or to act restricted to a narrow operation band.

For systems where the nonlinear terms cannot be ignored, however, a physical model very close to reality can be made. The solution is to use exact feedback linearization. In this case, it is possible to linearize the system and apply linear control methods. Unfortunately exact linearization cannot be used for noninvolutive systems, as they do not satisfy the aforementioned conditions.

To solve this problem, the approximate feedback linearization, formulated by Krener [3], has been adopted using differential geometry techniques.

The approximate feedback linearization may be considered a near equivalent version of the exact feedback linearization. On the other hand, it offers the possibility to control noninvolutive systems, which was not possible using exact linearization. This new approach has recently been used in several applications.

As an example to validate this technique, the inverted pendulum model (Figure 3) with its characteristic nonlinear dynamic and an unstable open loop will be used. It is a classic 
problem in control theory and is widely used as a benchmark for testing of control algorithms (adaptive control, robust control, fuzzy, etc.).

\subsection{Approximate Linearization Calculation}

The approximate feedback linearization has been applied in cases where exact linearization is not feasible or has not yielded satisfactory results [4]. This process consists of finding approximate output functions that satisfy the involutivity condition up to determined system order.

The basic idea is [5], given

$$
\dot{\mathbf{x}}=\mathbf{f}(\mathbf{x})+\mathbf{g}(\mathbf{x}) u
$$

where $\mathrm{x} \in R$.

Considering a state transformation in the control variable, we obtain

$$
\begin{gathered}
\mathbf{z}=W(\mathbf{x}), \\
u=\alpha(\mathbf{x})+\beta(\mathbf{x}) v,
\end{gathered}
$$

so that

$$
\dot{\mathbf{z}}=A \mathbf{z}(\mathbf{x})+b v+O^{p+1}(\widetilde{\mathbf{x}}, u)
$$

where $A=\partial \mathbf{f}\left(\mathbf{x}_{0}\right) / \partial \mathbf{x}, b=g\left(\mathbf{x}_{0}\right), \tilde{\mathbf{x}}=\mathbf{x}-\mathbf{x}_{0}, \mathbf{f}\left(\mathbf{x}_{0}\right)=0$.

We apply a state transformation in the control variable such as

$$
\begin{gathered}
\mathbf{z}(\mathbf{x})=\widetilde{\mathbf{x}}+\frac{1}{2} \mathbf{z}_{x x}\left(\mathbf{x}_{0}\right) \widetilde{\mathbf{x}}^{2}+O^{3}(\widetilde{\mathbf{x}}, u) \\
v(\mathbf{x}, u)=u+v_{x} \widetilde{\mathbf{x}}+\frac{1}{2} v_{x x} \widetilde{\mathbf{x}}^{2}+v_{x u} \widetilde{\mathbf{x}} u+O^{3}(\widetilde{\mathbf{x}}, u),
\end{gathered}
$$

where

$$
\mathbf{z}_{x x}=\left.\frac{\partial^{2} \mathbf{z}\left(\mathbf{x}_{0}\right)}{\partial \mathbf{x}^{2}}\right|_{x=x_{0}}, \quad v_{x x}=\left.\frac{\partial^{2} v(\mathbf{x}, u)}{\partial \mathbf{x}^{2}}\right|_{x=x_{0}}, \quad v_{x u}=\left.\frac{\partial^{2} v(\mathbf{x}, u)}{\partial \mathbf{x} \partial u}\right|_{x=x_{0}}
$$

Differentiating (4.4), we obtain

$$
\dot{\mathbf{z}}(\mathbf{x})=\dot{\mathbf{x}}+\mathbf{z}_{x x} \tilde{\mathbf{x}} \dot{\mathbf{x}}
$$

Substituting (4.1) in (4.7),

$$
\dot{\mathbf{z}}(\mathbf{x})=\mathbf{f}(\mathbf{x})+\mathbf{g}(\mathbf{x}) u+\mathbf{f}(\mathbf{x}) \mathbf{z}_{x x} \tilde{\mathbf{x}}+\mathbf{g}(\mathbf{x}) \mathbf{z}_{x x} \tilde{\mathbf{x}} u
$$


Expanding in a Taylor series,

$$
\dot{\mathbf{z}}(\mathbf{x})=A \widetilde{\mathbf{x}}+\mathbf{g}\left(\mathbf{x}_{0}\right) u+\left[\frac{\partial \mathbf{f}\left(\mathbf{x}_{0}\right)}{\partial \mathbf{x}} \mathbf{z}_{x x}+\frac{1}{2} \frac{\partial^{2} \mathbf{f}\left(\mathbf{x}_{0}\right)}{\partial \mathbf{x}^{2}}\right] \widetilde{\mathbf{x}}^{2}+\left[\frac{\partial \mathbf{g}\left(\mathbf{x}_{0}\right)}{\partial \mathbf{x}}+\mathbf{g}\left(\mathbf{x}_{0}\right) \mathbf{z}_{x x}\right] \tilde{\mathbf{x}} u+O^{3}(\widetilde{\mathbf{x}}, u)
$$

Substituting (4.4) and (4.5) in (4.3), we obtain

$$
\dot{\mathbf{z}}(\mathbf{x})=A \widetilde{\mathbf{x}}+\mathbf{g}\left(\mathbf{x}_{0}\right) u+\frac{1}{2}\left[\frac{\partial \mathbf{f}\left(\mathbf{x}_{0}\right)}{\partial \mathbf{x}} \mathbf{z}_{x x}+\mathbf{g}\left(\mathbf{x}_{0}\right) v_{x x}\right] \tilde{\mathbf{x}}^{2}+\mathbf{g}\left(\mathbf{x}_{0}\right) v_{x u} \tilde{\mathbf{x}} u+O^{3}(\widetilde{\mathbf{x}}, u) .
$$

Equalizing the second order term of (4.9) and (4.10) so that the unknown constants $\left(\mathbf{z}_{x x}, v_{x x}, v_{x u}\right)$ cancel out the second-order terms $\left(\tilde{\mathbf{x}}^{2}, \tilde{\mathbf{x}} u\right)$, we obtain

$$
\begin{gathered}
\frac{\partial \mathbf{f}\left(\mathbf{x}_{0}\right)}{\partial \mathbf{x}} \mathbf{z}_{x x}+\frac{\partial^{2} \mathbf{f}\left(\mathbf{x}_{0}\right)}{\partial \mathbf{x}^{2}}-\mathbf{g}\left(\mathbf{x}_{0}\right) v_{x x}=0, \\
\frac{\partial \mathbf{g}\left(\mathbf{x}_{0}\right)}{\partial \mathbf{x}}+\mathbf{g}\left(\mathbf{x}_{0}\right) \mathbf{z}_{x x}-\mathbf{g}\left(\mathbf{x}_{0}\right) v_{x u}=0 .
\end{gathered}
$$

Thus, there is an approximate output $W_{1}(\mathbf{x})$, such as the set of vectors $\left\{\mathbf{g}, a d_{\mathbf{f}} \mathbf{g}, \ldots, a d_{\mathbf{f}}^{\mathrm{n}-2} \mathbf{g}\right\}$ are involutive until certain order $p$ [6]. In other words, the gradient $W_{1}(\mathbf{x})$ multiplied by the Lie bracket of $\mathbf{f}$ and $\mathbf{g}$ is equal to the superior terms $p+1$. The following equation illustrates this:

$$
\nabla W_{1}(\mathbf{x}) a d_{\mathrm{f}}^{i} \mathbf{g}=O^{p+1}(\tilde{\mathbf{x}}), \quad i=0,1,2, \ldots, n-2 .
$$

In order to find vector $W_{1}$, it is necessary to expand the involutive distribution $D_{I}=$ $\left\{\mathbf{g}, a d_{\mathbf{f}} \mathbf{g}, \ldots, a d_{\mathbf{f}}^{n-2} \mathbf{g}\right\}$ in a Taylor series on an equilibrium point $\mathbf{x}_{0}$. The expansion of the involutive distribution in $\mathbf{x}_{0}$ can be represented through the following equation:

$$
D_{I}(\mathbf{x})=D_{I}\left(\mathbf{x}_{0}\right)+\sum_{k-1}^{n} \frac{\partial D_{I}\left(\mathbf{x}_{0}\right)}{\partial \mathbf{x}_{k}} \tilde{\mathbf{x}}_{k}+\frac{1}{2} \sum_{k, i-1}^{n} \frac{\partial^{2} D_{I}\left(\mathbf{x}_{0}\right)}{\partial \mathbf{x}_{k} \partial \mathbf{x}_{i}} \tilde{\mathbf{x}}_{k} \tilde{\mathbf{x}}_{i}+\mathrm{O}^{3}(\widetilde{\mathbf{x}}),
$$

where $\widetilde{\mathbf{x}}_{k}=\mathbf{x}_{k}-\mathbf{x}_{0}$ and $\tilde{\mathbf{x}}_{i}=\mathbf{x}_{i}-\mathbf{x}_{0}$

This expansion is carried out in order to obtain an involutivity until a certain order $p$, where $p$ indicates the order in which the involutive distribution is expanded in a Taylor series to satisfy the involutivity condition. Therefore, the calculation of the approximate output function $W_{1}$ is done through the equation

$$
\left[D_{0}+D_{1}+\cdots+D_{p}\right]^{T} \nabla W_{1}=0_{n-1 \times 1}
$$


where,

$$
\begin{aligned}
& D_{0}=D_{I}\left(\mathbf{x}_{0}\right), \\
& D_{1}=\sum_{k=1}^{n} \frac{\partial D_{I}\left(\mathbf{x}_{0}\right)}{\partial \mathbf{x}_{k}} \widetilde{\mathbf{x}}_{k}, \\
& \vdots \\
& \mathbf{D}_{p}=\frac{1}{p !} \sum_{k, l, \ldots, z=1}^{n} \frac{\partial^{p} \mathbf{D}_{I}\left(\mathbf{x}_{0}\right)}{\partial \mathbf{x}_{k} \partial \mathbf{x}_{l} \cdots \partial \mathbf{x}_{z}} \widetilde{\mathbf{x}}_{k} \widetilde{\mathbf{x}}_{l} \cdots \widetilde{\mathbf{x}}_{z} .
\end{aligned}
$$

Once $W_{1}(\mathbf{x})$ is known, the equation of the remaining state is given by

$$
\lambda_{k}(\mathbf{x})=L_{\mathbf{f}}^{k-1} W_{1}(\mathbf{x})
$$

And the input transformation

$$
v=L_{\mathrm{f}}^{k} W_{1}(\mathbf{x})+L_{\mathrm{g}} L_{\mathrm{f}}^{k-1} W_{1}(\mathbf{x}) u
$$

The state equations are represented as

$$
\begin{gathered}
\dot{W}_{i}=W_{i+1}+\mathrm{O}^{p+1}(\tilde{\mathbf{x}}, u), \\
\dot{W}_{n}=v+\mathrm{O}^{p+1}(\tilde{\mathbf{x}}, u) .
\end{gathered}
$$

It is important to emphasize that the choice for the representation of the linearized system was based on the Brunovski canonical form.

\subsection{Inverted Pendulum Linearization}

The proposed inverted pendulum consists of a rigid rod of negligible weight, attached to a mobile base called car and with a fixed mass at the other end. The inferior part of the pendulum rod is in direct contact with the car, which aims to keep the rod in the upright position according to the movement performance of the car control position. In the context of this paper, a pendulum that performs its movement in only one plane was conidered. In other words, it has only one degree of freedom.

We consider the following nonlinear function:

$$
\dot{\mathbf{x}}=\mathbf{f}(\mathbf{x})+\mathbf{g}(\mathbf{x}) u,
$$


where $x_{1}=x, x_{2}=\dot{x}_{1}, x_{3}=\theta$, and $x_{4}=\dot{\theta}$, and the movement equation may be described as [7]

$$
\begin{gathered}
\mathbf{f}(\mathbf{x})=\left[\begin{array}{c}
x_{2} \\
\frac{\sin \left(x_{3}\right) x_{4}^{2}-g \sin \left(x_{3}\right) \cos \left(x_{3}\right)}{2-\cos \left(x_{3}\right)^{2}} \\
x_{4} \\
\frac{-\sin \left(x_{3}\right) \cos \left(x_{3}\right) x_{4}^{2}+2 g \sin \left(x_{3}\right)}{2-\cos \left(x_{3}\right)^{2}}
\end{array}\right], \\
\mathbf{g}(\mathbf{x})=\left[\begin{array}{c}
0 \\
\frac{1}{2-\cos \left(x_{3}\right)^{2}} \\
0 \\
\frac{-\cos \left(x_{3}\right)}{2-\cos \left(x_{3}\right)^{2}}
\end{array}\right]
\end{gathered}
$$

For the involutive order 1 , there should be a $W_{1}$, not trivial, that satisfies the equation below [8]:

$$
\nabla W_{1} \mathbf{D}_{0}=0,
$$

where

$$
\mathbf{D}_{0}=\left[\begin{array}{ccc}
0 & -1 & 0 \\
1 & 0 & g \\
0 & 1 & 0 \\
-1 & 0 & -2 g
\end{array}\right]
$$

resulting in

$$
\nabla W_{1}=\left[\begin{array}{llll}
1 & 0 & 1 & 0
\end{array}\right]^{T}
$$

integrating

$$
W_{1}=x_{1}+x_{3}
$$

Once $W_{1}$ has been calculated, the remaining states and the control variable are obtained from [6].

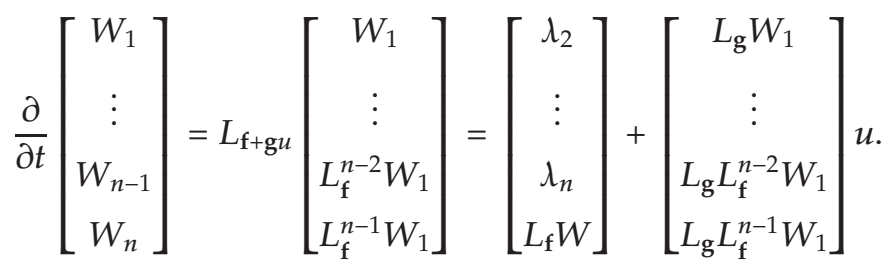

The last column on the right side represents the liberalized system neglected terms. 
The input variable is given by

$$
u=\alpha(\mathbf{x})+\beta(\mathbf{x}) v
$$

where

$$
\begin{aligned}
& \alpha(\mathbf{x})=\frac{-L_{\mathrm{f}}^{n} W_{1}}{L_{\mathrm{g}} L_{\mathrm{f}}^{n-1} W_{1}}, \\
& \beta(\mathbf{x})=\frac{1}{L_{\mathrm{g}} L_{\mathrm{f}}^{n-1} W_{1}},
\end{aligned}
$$

resulting in system approximately linear given by

$$
\frac{\partial}{\partial t}\left[\begin{array}{c}
W_{1} \\
\vdots \\
W_{n-1} \\
W_{n}
\end{array}\right]=\left[\begin{array}{llll}
0 & 1 & 0 & 0 \\
0 & 0 & 1 & 0 \\
0 & 0 & 0 & 1 \\
0 & 0 & 0 & 0
\end{array}\right]\left[\begin{array}{c}
W_{1} \\
\vdots \\
W_{n-1} \\
W_{n}
\end{array}\right]+\left[\begin{array}{l}
0 \\
0 \\
0 \\
1
\end{array}\right] v
$$

where

$$
\begin{gathered}
\dot{W}_{i}=W_{i+1}+O^{p+1}(\tilde{\mathbf{x}}, u), \\
\dot{W}_{n}=v+O^{p+1}(\widetilde{\mathbf{x}}, u) .
\end{gathered}
$$

\section{Simulation Results}

In the simulation, a comparison of a controller for an inverted pendulum using the Taylor linearization and the approximate feedback linearization techniques was carried out. The pole placement control technique was used, where the close loop poles in both linearizations were placed in $s=-1, s=-2, s=-1+j$, and $s=-1-j$. It can be seen from Figures 4 and 5 that the controller based on the approximate feedback linearization performed better for the initial positions $x_{1}(0)=x_{2}(0)=x_{4}(0)=0, x_{3}(0)=40^{\circ}$, and $x_{3}(0)=30^{\circ}$. From the simulation results, it can be seen that for the Taylor linearization, the controller managed to stabilize the inverted pendulum for $x_{3}(0) \leq 41^{\circ}$, while with the approximate linearization, the controller managed the stabilization for $x_{3}(0) \leq 53^{\circ}$, which represents an increase in the inverted pendulum operation region.

The simulation shows the effectiveness of the proposed controller. Another important factor was the response of the controller as regards its position. According to Figures 6 and 7, the displacement of the inverted pendulum was less for the controller based on the approximate feedback linearization.

Another simulation was performed increasing the weight of the fixed mass attached to the car which contains the weight five times. 


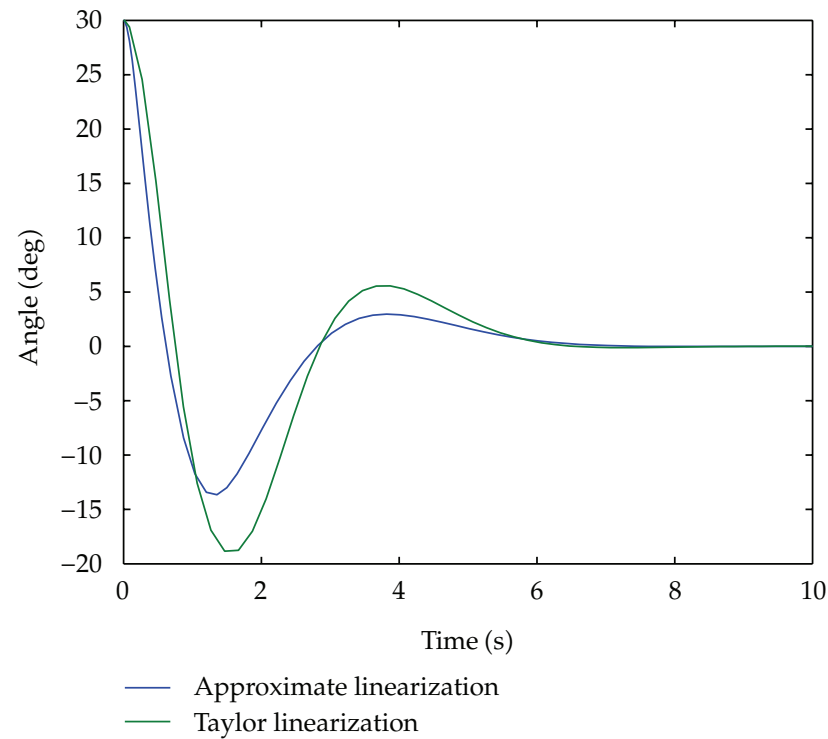

Figure 4: Inverted pendulum for $x_{3}(0)=30^{\circ}$.

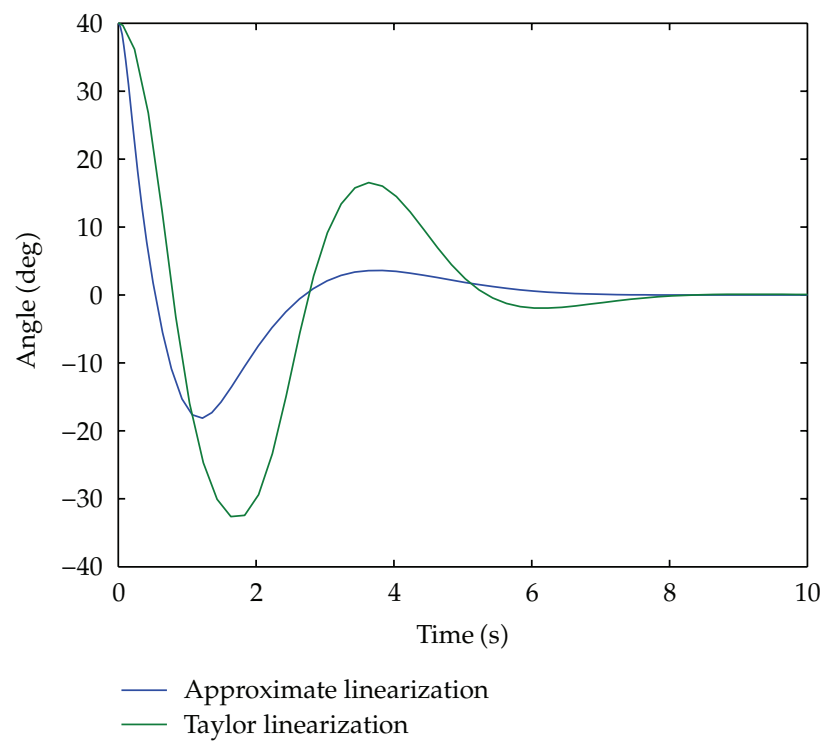

Figure 5: Inverted pendulum for $x_{3}(0)=40^{\circ}$.

\section{Conclusions}

This work describes feedback linearization techniques used as a tool for differential geometry calculus. The existing restrictions to the use of the exact input-state feedback linearization technique were analysed, and the impossibility of using exact linearization in involutive systems was observed. As a solution to this, we proposed approximate linearization. 


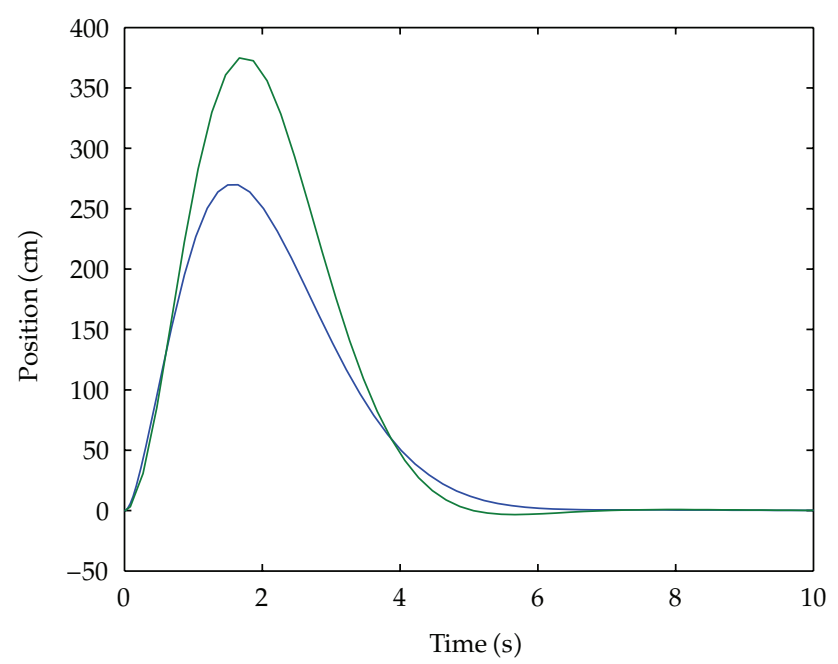

- Approximate linearization

— Taylor linearization

Figure 6: Inverted pendulum for $x_{3}(0)=30^{\circ}$.

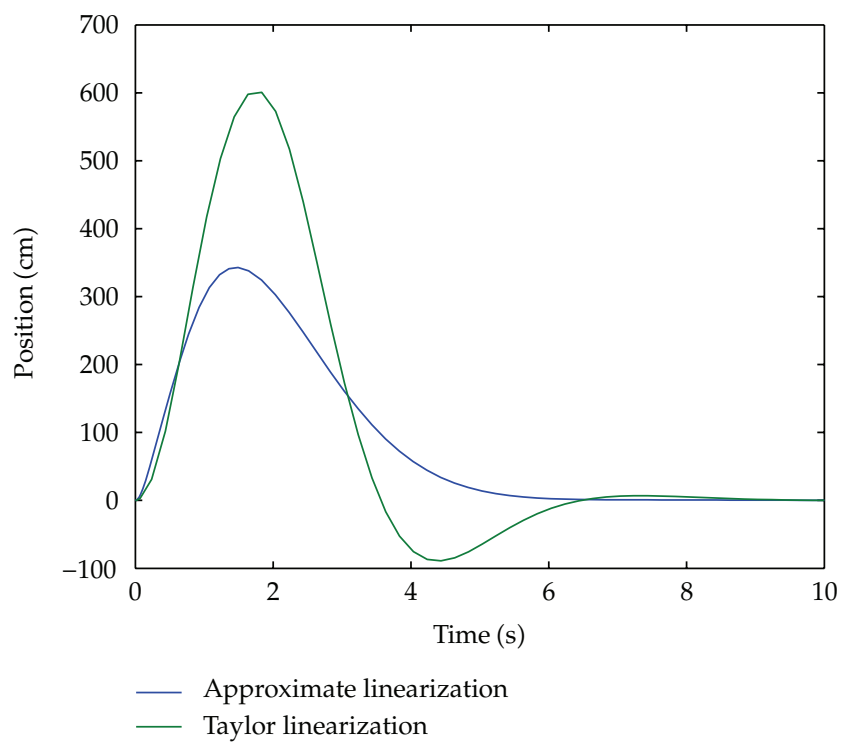

Figure 7: Inverted pendulum for $x_{3}(0)=40^{\circ}$.

To illustrate the problem of exact linearization, a simple pendulum was used, where the calculation of the input variable as well as the state transformations were performed using differential geometry as the main calculus tool.

In the approximate linearization technique, an inverted pendulum was used, as it is an example of great interest. The oscillating behaviour of the inverted pendulum rod reproduces the stabilization problem common in situations such as the trajectory of a projectile or the movement of a satellite. In addition, it is an unstable in open-loop, nonlinear, noninvolutive, 


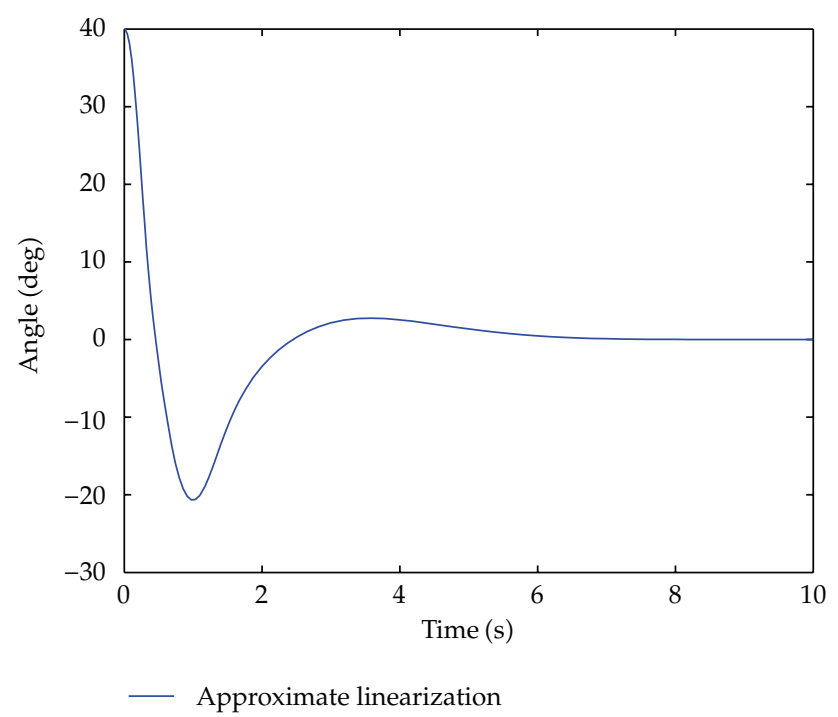

Figure 8: Inverted pendulum for $x_{3}(0)=40^{\circ}(m=5 \mathrm{~kg})$.

and an underactuated system, which makes it attractive for application in advanced control techniques.

As a demonstration of the effectiveness of the method, a simulation was carried out to compare the Taylor linearization with the approximate feedback linearization, with the latter technique increasing the inverted pendulum operation region.

According to the results obtained with a five-kilogram weight, shown in Figure 8, on the top of the inverted pendulum flexible rod, it can be inferred that there is the possibility possess a good robustness.

Noninvolutivity, an important detail that prevents the application of the exact feedback linearization technique, was solved through coordinate transformation. This new state space, in turn, is involutive, enabling, therefore, exact linearization. In other words, as this coordinate transformation generates small errors, the linearization is no longer exact, making it an approximate linearization. However, it has been noted that despite these errors, the results indicate that the technique achieved a superior performance when compared to the Taylor linearization.

\section{References}

[1] J. J. Slotine and L. Weiping, Applied Nonlinear Control, Prentice-Hall, Englewood Cliffs, NJ, USA, 1991.

[2] J. Bronislaw, "Introduction to geometric nonlinear control; controllability and lie bracket," in Mathematical Control Theory, Lectures Notes of a Minicourse, pp. 107-168, Abdus Salam International Center Theoret Physics, Trieste, Italy, 2002.

[3] A. J. Krener, "Approximate linearization by state feedback and coordinate change," Systems E Control Letters, vol. 5, no. 3, pp. 181-185, 1984.

[4] S. Renou and S. Saydy, "Real time control of an inverted pendulum based on approximate linearization," in Proceedings of the Canadian Conference on Electrical and Computer Engineering (CCECE '96), vol. 2, pp. 502-504, May 1996.

[5] N. Bedrossian, Nonlinear control using linearizing transformations, Ph.D. thesis, Department of Mechanical Engineering, Massachusetts Institute of Technology, Cambridge, Mass, USA, 1991. 
[6] N. S. Bedrossian, "Approximate feedback linearization: The cart-pole example," in Proceedings of the IEEE International Conference on Robotics and Automation, vol. 3, pp. 1987-1992, 1992.

[7] J. Deutscher and C. Schmid, "A state space embedding approach to approximate feedback linearization of single input nonlinear control systems," International Journal of Robust and Nonlinear Control, vol. 16, no. 9, pp. 421-440, 2006.

[8] L. Guzzella and A. Isidori, "On approximate linearization of nonlinear control systems," International Journal of Robust \& Nonlinear Control, vol. 3, no. 3, pp. 261-276, 1993. 


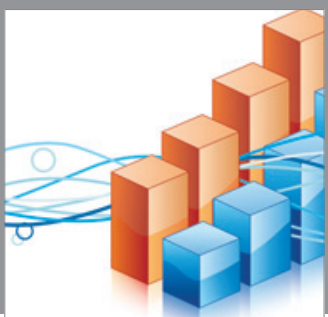

Advances in

Operations Research

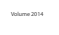

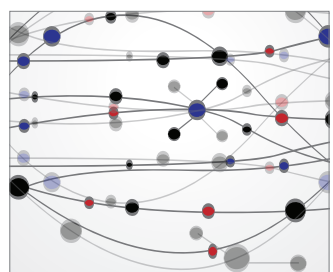

\section{The Scientific} World Journal
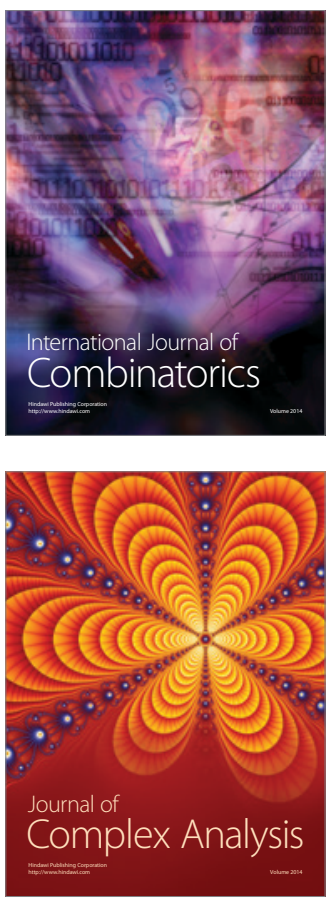

International Journal of

Mathematics and

Mathematical

Sciences
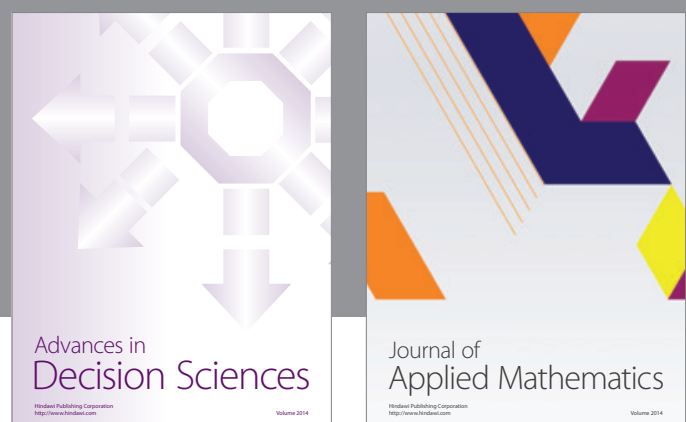

Journal of

Applied Mathematics
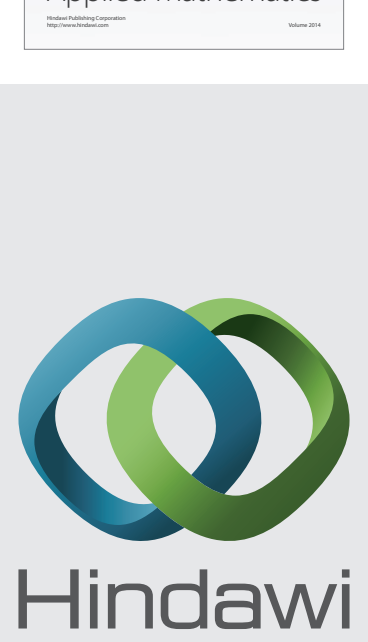

Submit your manuscripts at http://www.hindawi.com
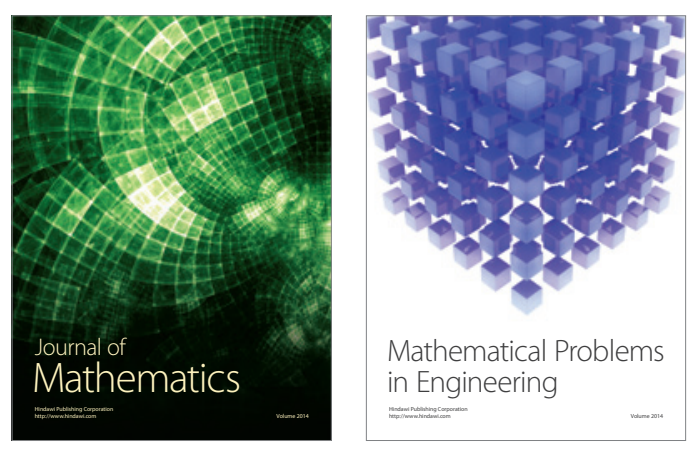

Mathematical Problems in Engineering
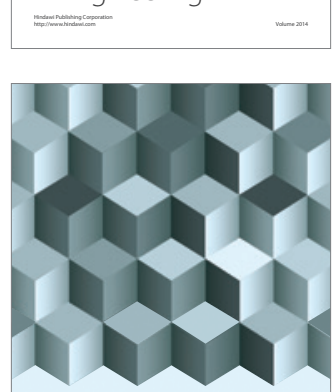

Journal of

Function Spaces
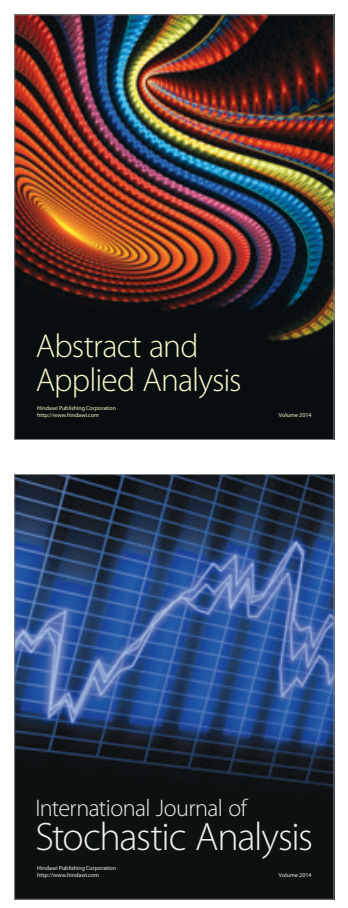

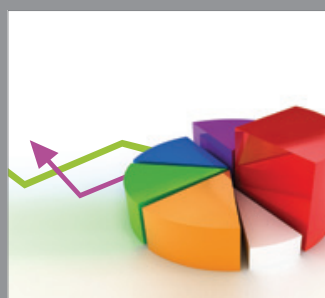

ournal of

Probability and Statistics

Promensencen
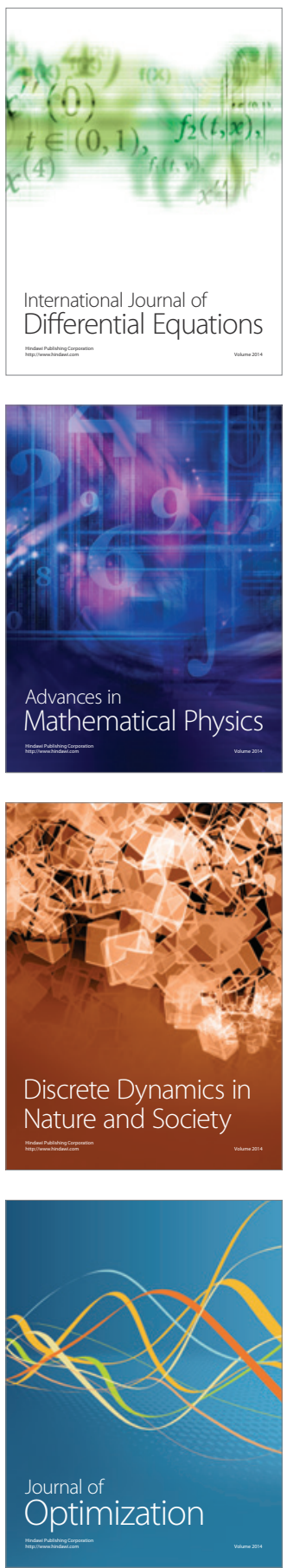